\title{
Charge Transport in Spiro-OMeTAD Investigated through Space-Charge-Limited Current Measurements
}

\author{
Jason A. Röhr, ${ }^{1,2, \dagger}$ Xingyuan Shi, ${ }^{1}$ Saif A. Haque, ${ }^{2}$ Thomas Kirchartz, ${ }^{3,4}$ and Jenny Nelson ${ }^{1, *}$ \\ ${ }^{1}$ Department of Physics \& Centre for Plastic Electronics, Imperial College London, \\ South Kensington Campus, London SW7 2AZ, United Kingdom \\ ${ }^{2}$ Department of Chemistry \& Centre for Plastic Electronics, Imperial College London, \\ South Kensington Campus, London SW7 2AZ, United Kingdom \\ ${ }^{3}$ IEK5-Photovoltaics, Forschungszentrum Jülich, 52425 Jülich, Germany \\ ${ }^{4}$ Faculty of Engineering and CENIDE, University of Duisburg-Essen, Carl-Benz-Strasse 199, \\ 47057 Duisburg, Germany
}

(Received 17 July 2017; revised manuscript received 20 December 2017; published 12 April 2018)

\begin{abstract}
Extracting charge-carrier mobilities for organic semiconductors from space-charge-limited conduction measurements is complicated in practice by nonideal factors such as trapping in defects and injection barriers. Here, we show that by allowing the bandlike charge-carrier mobility, trap characteristics, injection barrier heights, and the shunt resistance to vary in a multiple-trapping drift-diffusion model, a numerical fit can be obtained to the entire current density-voltage curve from experimental space-charge-limited current measurements on both symmetric and asymmetric 2, 2', 7, $7^{\prime}$-tetrakis $(N, N$-di-4-methoxyphenylamine)$9,9^{\prime}$-spirobifluorene (spiro-OMeTAD) single-carrier devices. This approach yields a bandlike mobility that is more than an order of magnitude higher than the effective mobility obtained using analytical approximations, such as the Mott-Gurney law and the moving-electrode equation. It is also shown that where these analytical approximations require a temperature-dependent effective mobility to achieve fits, the numerical model can yield a temperature-, electric-field-, and charge-carrier-density-independent mobility. Finally, we present an analytical model describing trap-limited current flow through a semiconductor in a symmetric single-carrier device. We compare the obtained charge-carrier mobility and trap characteristics from this analytical model to the results from the numerical model, showing excellent agreement. This work shows the importance of accounting for traps and injection barriers explicitly when analyzing current density-voltage curves from space-charge-limited current measurements.
\end{abstract}

DOI: 10.1103/PhysRevApplied.9.044017

\section{INTRODUCTION}

In recent years, there has been a great deal of interest in understanding the charge-carrier transport of weakly doped or undoped semiconducting thin films based on disordered molecular materials such as $\pi$-conjugated small molecules and polymers [1-4]. Studying space-charge-limited currents (SCLCs) in single-carrier devices is an important means to understand electron and hole transport in such semiconductors. Although there have been many earlier reports on the charge-carrier transport of molecular materials using SCLCs, the vast majority of these rely on using the Mott-Gurney (MG) law [5], which is not necessarily suitable for the analysis since this law relies on idealized and trap-free semiconductors in devices with contacts which are not too injection limiting. In contrast, many

\footnotetext{
*Corresponding author. jenny.nelson@imperial.ac.uk

Corresponding author. jason.rohr@yale.edu
}

molecular materials contain charge-carrier traps, and single-carrier devices usually have some contact asymmetry due to the relative difficulty of ensuring truly identical and ideal contacts on both sides of the semiconducting thin film whose mobility has to be measured.

Analytical equations have been derived to describe charge transport when either traps [6-8] or a built-in voltage resulting from contact asymmetry is present $[9,10]$. Mark and Helfrich derived an equation describing SCLCs in the drift-dominated voltage regime when the charge transport of the semiconductor was limited by energetic disorder due to the localization of charge carriers in exponential tails in the band gap [8]. Fischer et al. have, however, pointed out that this so-called Mark-Helfrich equation is imprecise in estimating the trap characteristics since the equation does not take diffusion of charge carriers into account [11], which may affect the current density in the Mott-Gurney regime when charge carriers are localized in exponential tails. For that reason, a good analytical model describing space-charge-limited charge transport in 
disordered semiconductor devices does not currently exist. Moreover, no analytical model exists that describes the common practical situation where both a large built-in voltage and traps are present. As a result, less suitable models, such as the MG law, are often used to analyze the experimental data, despite evidence for both trapping and built-in voltages. While more sophisticated drift-diffusion simulations can be used to analyze the experimental data more accurately, only a small number of publications adopt that approach $[3,12-14]$.

It is common within the organic semiconductor community to model the total density of states (DOS) using a Gaussian distribution (alternative approaches using master equations have also been used $[15,16])$. Hopping charge transport in such a Gaussian DOS leads to a dependence of the (effective) mobility on the temperature, electric field, and charge-carrier density that has been parametrized by Pasveer et al. and subsequently used in a series of papers describing unipolar transport in organic semiconductors $[17,18]$. However, it has previously been shown that exponential tails (which is a good approximation of a Gaussian in a limited energy range) or even more complex shapes of the DOS are sometimes required to describe certain aspects of the physics of organic semiconductor devices $[3,12-14,19,20]$. For instance, molecular dynamics and tight-binding simulations on P3HT lead to a DOS that is neither a pure Gaussian nor a pure exponential tail but rather a combination of both [21]. Thus, there is value in having models that allow us to change the shape of the DOS without starting with the process of parametrizing mobility as a function of the temperature, electric field, and chargecarrier density as previously done, but rather account for these effects directly in the drift-diffusion model through the trap characteristics and interface statistics.

Here, we show how using drift-diffusion simulations improves the accuracy and physical interpretation of the determined mobility relative to traditional analytical approaches. We show that the obtained mobility depends only on the temperature, electric field, and charge-carrier density through the trap and injection characteristics. In addition, we derive a simple analytical description of the current density, which can be used to determine the trap characteristics of a symmetric single-carrier device with high precision when the semiconductor has an exponential trap distribution by examination of the low-voltage current regime rather than from the current in the intermediate Mott-Gurney regime. A small molecule system commonly used as a hole-selective interlayer for dye-sensitized solar cells and organometallic perovskite cells, spiro-OMeTAD, is used for the study. Spiro-OMeTAD is chosen based on the expectation that its amorphous microstructure is largely insensitive to the layer thickness allowing for a thickness series to be performed. It is shown that when hole transport in spiro-OMeTAD is characterized using either the driftdiffusion solver approach (including exponential tails) or by using the analytical model proposed herein, the bandlike hole mobility is determined to be both temperature, field, and charge-carrier-density independent and substantially higher than the effective mobility that has been previously extracted using the more simple Mott-Gurney law [22].

\section{BACKGROUND THEORY}

First, we discuss the concept of single-carrier devices, along with injection barriers and built-in voltages in such devices, and then we discuss some common analytical approximations that have been used to analyze SCLC data.

\section{A. Single-carrier devices}

When the selected electrodes form contacts both to the conduction-band edge or both to the valence-band edge of an intrinsic (or weakly doped) semiconductor, the currentvoltage relation is governed by a single carrier type, with the charge-carrier species determined by the charge selectivity of the contact [an electron-only device is shown in Fig. 1(a)]. When a voltage $V$ is applied across such a singlecarrier device, excess charge carriers of the same species as the ones present at equilibrium are injected, and the current is space-charge limited [Fig. 1(b)]. For an electron-only device, if the work functions (WFs) of the contacts are larger than the electron affinity of the semiconductor, injection barriers will arise [shown as $q \phi_{\text {inj }}$ and $q \phi_{\text {ext }}$ in Fig. 1(a)], and if these values are different (and nonzero), a built-in voltage will be present across the device $\left(q V_{\mathrm{bi}}=q \phi_{\mathrm{ext}}-q \phi_{\mathrm{inj}}\right)$, which gives rise to large diffusion currents when $V<V_{\text {bi }}$ [Fig. 1(c)].

\section{B. The Mott-Gurney law}

The most commonly used method for fitting data obtained from single-carrier devices is to use the MG law. The MG law describes a space-charge-limited drift current in an idealized single-carrier device made from a trap-free and undoped semiconductor in the intermediate voltage regime and in the limit of barrier-free injection [Fig. 1(b)] [[5,6,23]]. The MG law is given by

$$
J=\frac{9}{8} \mu \varepsilon_{r} \varepsilon_{0} \frac{V^{2}}{L^{3}},
$$

where $\mu$ is the charge-carrier mobility for either electrons or holes ( $\mu_{n}$ for electrons and $\mu_{h}$ for holes), $\varepsilon_{r}$ is the static relative permittivity, $\varepsilon_{0}$ is the permittivity of free space, $V$ is the applied voltage, and $L$ is the thickness of the semiconductor layer. However, organic semiconducting materials are rarely trap-free, rendering the simple MG theory improper for describing charge transport for most realistic cases. It is, however, still common to assume an effective mobility $\mu_{\text {eff }}$ in the case where the MG law is used even though the semiconductor contains traps. This effective mobility is usually defined as $[11,24]$ 
(a)

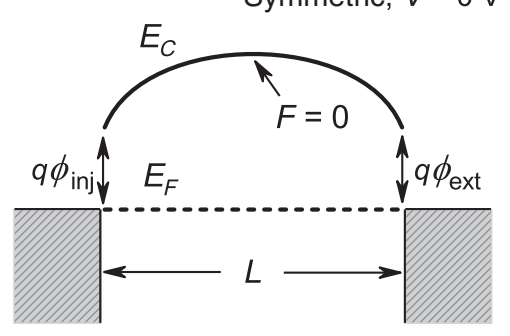

(c)

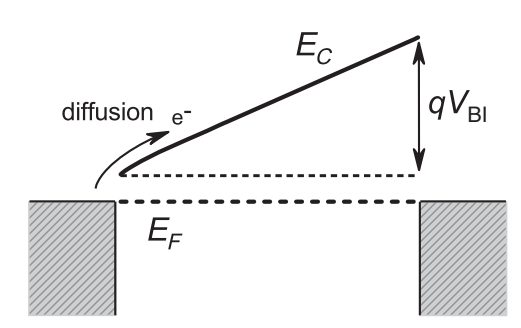

(b)

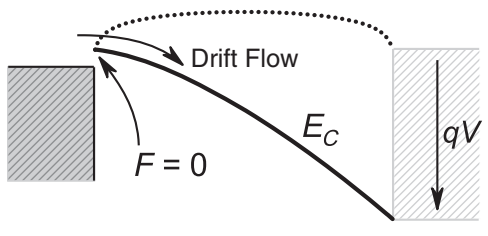

Symmetric, $V>0 \mathrm{~V}$

(d)

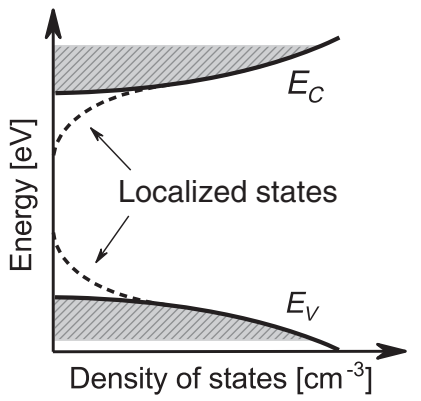

FIG. 1. Schematic of the energy-level diagrams of (a) symmetric electron-only device at thermodynamic equilibrium, where $q \varphi_{\text {inj }}$ and $q \varphi_{\text {ext }}$ represent the injection and extraction barrier heights, respectively, $E_{C}$ and $E_{V}$ are the conduction- and valence-band edge, respectively, $E_{F}$ is the Fermi level of the semiconductor at thermal equilibrium, and $F$ is the electric field. (b) Symmetric electron-only device with enough applied voltage to assume drift-dominant transport (Mott-Gurney regime). (c) Asymmetric electron-only device at equilibrium showing the energy barrier arising from a built-in voltage $q V_{\mathrm{bi}}=q \varphi_{\mathrm{ext}}-q \varphi_{\mathrm{inj}}$. (d) Sketch of the total DOS including exponential tail states (the depth of the tails are given by their respective characteristic energies). Electron transport is shown for simplicity (hole transport is completely analogous). In (b) and (c), forward injection of electrons from the left-hand side is assumed.

$$
\mu_{\text {eff }}=\mu_{\text {band }} \frac{\left\langle\rho_{\text {free }}\right\rangle}{\left\langle\rho_{\text {total }}\right\rangle},
$$

where $\mu_{\text {band }}$ is the bandlike mobility of the semiconductor [which is approximately equal to $\mu$ in Eq. (1) in an ideal case], $\left\langle\rho_{\text {free }}\right\rangle=L^{-1} \int_{0}^{L} \rho_{\text {free }}(x) d x$ is the spatial average of the free charge-carrier density, and $\left\langle\rho_{\text {total }}\right\rangle=L^{-1} \int_{0}^{L}\left[\rho_{\text {free }}(x)+\right.$ $\left.\rho_{\text {trapped }}(x)\right] d x$ is the total charge-carrier density across the thickness of the semiconductor. Since the effective mobility in Eq. (2) is defined from the ratio of the free to total chargecarrier density, the effective mobility is usually electric field and temperature dependent. However, the band mobility is not inherently dependent on either of these quantities [19]. Note that this definition of the effective mobility does not account for nonideal injection.

\section{The Mark-Helfrich equation}

One of the few analytical equations which describes SCLC charge transport in a semiconductor with traps, assumed to be distributed as exponential tails of states in the DOS, is called the Mark-Helfrich equation [6,8]. For an electron-only device, it is given by

$$
J=q^{1-l} \mu_{n} N_{\text {eff }}\left\{\frac{\varepsilon_{r} \varepsilon_{0} l}{N_{t}(l+1)}\right\}^{l}\left\{\frac{2 l+1}{l+1}\right\}^{l+1} \frac{V^{l+1}}{L^{2 l+1}},
$$

where $q$ is the elementary charge, $l=E_{\mathrm{ch}} / k_{B} T$, with $E_{\mathrm{ch}}$ being the characteristic energy of the exponential tail [see Fig. 1(d) and the Supplemental Material [25] Eqs. (S30) and (S31)], $N_{\text {eff }}$ is the effective density of states, $N_{t}$ is the trap density (per unit volume), and $k_{B} T$ is the thermal energy. Equation (3) predicts that exponential tail states in the band gap give rise to a stronger power-law dependence of voltage on current than expected from the MG law in the intermediate-voltage regime. However, it was recently shown that this equation is not accurate since it fails to account for diffusion currents, which can make a significant contribution to the total current, especially when traps are present [11].

\section{Built-in voltages}

In addition to assuming the semiconductor to be trapfree, the MG law also assumes that there are negligible energy barriers for both injection $\left(q \phi_{\text {inj }}\right)$ and extraction $\left(q \phi_{\text {ext }}\right)$ from the metal contact into the semiconductor. This criterion can, however, rarely be met in real experimental cases, and a finite injection barrier can have a significant influence on the probed charge-transport behavior. With $q \phi_{\text {inj,ext }}=0 \mathrm{eV}$ being assumed, the MG law, therefore, also implicitly requires that there exists no built-in voltage $V_{\mathrm{bi}}$ across the device arising from a difference in the WFs, and, hence, the injection barrier heights $V_{\mathrm{bi}}=q \phi_{\mathrm{ext}}-q \phi_{\text {inj }}$ [Fig. 1(c)] [5], a feature which is often difficult to achieve 
from a practical device fabrication perspective. The limited choice of contact materials available to match very deep ( $>\sim 6 \mathrm{eV}$ below the vacuum level) or very shallow $(<\sim 2 \mathrm{eV}$ below the vacuum level) transport levels often results in contacts with poor injection properties. A common hole-selective material for organic optoelectronic devices is the conductive polymer blend, poly(3,4-ethylenedioxythiophene) polystyrene sulfonate (PEDOT:PSS). The literature values for the WF of this conductive polymer range between 4.8 and $5.2 \mathrm{eV}[26,27]$. With many semiconducting polymers and small molecules having their highest occupied molecular orbital (HOMO) levels lying much deeper than $5.2 \mathrm{eV}$ below the vacuum level, this interlayer material will not form a perfectly injecting contact with such materials. Another popular hole-selective material is $\mathrm{MoO}_{3}$, with literature values for the WF ranging between 6 and $6.9 \mathrm{eV}[28,29]$. Sandwiching a deep HOMO ( $>6 \mathrm{eV}$ below the vacuum level) material between these two contact materials, for example, will allow for Ohmic injection from $\mathrm{MoO}_{3}$ into the $\mathrm{HOMO}$ level, whereas the PEDOT:PSS will form an injection-limited contact, while at the same time resulting in the formation of a $V_{\mathrm{bi}}$ across the device due to the difference in the contact WFs [Fig. 1(c)]. Because of lack of contact materials, it is common to measure SCLCs on asymmetric single-carrier devices, since such devices are more realistic to fabricate than symmetric single-carrier devices [Fig. 1(c)]. However, this $V_{\mathrm{bi}}$ will greatly affect the current density-voltage $(J-V)$ curves at low and intermediate bias voltages until the internal voltage is overcome $[9,10]$.

In order to correct for the built-in voltage, an effective applied voltage $V_{\text {eff }}=V-V_{\mathrm{bi}}$, where the $V_{\mathrm{bi}}$ is used as a fitting parameter in the MG law (or other analytical equations of variant types, such as the $\mathrm{MH}$ equation or the Murgatroyd equation [30]), is commonly used [31,32]. This indirect approach is, however, rather uncertain, since the band diagram at $V=0 \mathrm{~V}$ for a symmetric device and $V=V_{\text {bi }}$ for an asymmetric device are not necessarily similar. At a significant applied bias such that the built-in voltage is overcome, and if, hypothetically, the value of $V_{\mathrm{bi}}$ is exactly known, then using this effective voltage approach is a good approximation. The $V_{\mathrm{bi}}$ is, however, rarely known precisely in advance, and it is sometimes estimated by shifting the voltage axis to a regime where $J$ varies with $V^{2}$, which can eventually lead to misinterpretation of carrier mobility values, especially when traps are present.

Analytical drift-diffusion equations have been derived to account for built-in voltages directly in intrinsic singlecarrier devices at low voltages directly, such as $[9,10]$

$$
J=\frac{q \mu N_{\text {eff }}\left(\phi_{\text {ext }}-\phi_{\text {inj }}-b-V\right)\left\{\exp \left(\frac{q V}{k_{B} T}\right)-1\right\}}{L \exp \left(\frac{q \phi_{\text {inj }}}{k_{B} T}\right) \exp \left(\frac{q b}{k_{B} T}\right)\left\{\exp \left(\frac{q \phi_{\mathrm{ext}}-q \phi_{\text {inj }}-q b}{k_{B} T}\right)-\exp \left(\frac{q V}{k_{B} T}\right)\right\}},
$$

where $b$ is the voltage reduction due to band bending at the injecting interface [the derivation of Eq. (4) is shown in the Supplemental Material [25] and in Ref. [10] ]. Assuming the $V_{\mathrm{bi}}$ is large enough so that band bending at the interface is negligible $b=0$ and the injection barrier height at the injection point is zero $q \phi_{\text {inj }}=0 \mathrm{eV}$, Eq. (4) is reduced to a simpler form

$$
J=\frac{q \mu N_{\mathrm{eff}}\left(V_{\mathrm{bi}}-V\right)\left\{\exp \left(\frac{q V}{k_{B} T}\right)-1\right\}}{L\left\{\exp \left(\frac{q V_{\mathrm{bi}}}{k_{B} T}\right)-\exp \left(\frac{q V}{k_{B} T}\right)\right\}},
$$

which is a useful equation for determining the built-in voltage of a single-carrier device [9]. Equation (5) describes the current density of an asymmetric intrinsic semiconductor device for all $V<V_{\mathrm{bi}}$. However, it cannot describe the situation where traps are present. Even though analytical models exist to describe both a built-in voltage and traps separately, no analytical model exists to describe the common practical situation featuring both traps and a large built-in voltage.

\section{E. Low-voltage regime}

The final set of analytical models presented here applies to the low-voltage regime (from a symmetric single-carrier device), where, in practice, a linear dependence of $J$ on $V$ is often observed. It has previously been shown that linear currents observed in SCLC $J-V$ curves are due to one of, or a combination of, the following effects: flow of equilibrium charge carriers at low voltages in symmetric single-carrier devices (the moving-electrode equation) [33-35], a large increase of the charge-carrier density away from the equilibrium value due to doping $[35,36]$, or bulk saturation of charge carriers inside the device either at high bias or with large injection barriers [37].

In the absence of traps, doping, and a built-in voltage, the current density at low voltages is given by the movingelectrode (ME) equation [33,34,38-40],

$$
J=4 \pi^{2} \frac{k_{B} T}{q} \mu \varepsilon_{r} \varepsilon_{0} \frac{V}{L^{3}} .
$$

Linear currents can, however, also arise at low voltages in single-carrier devices through shunts due to lowresistance pathways through the semiconducting film. This linear current density is given by Ohm's law as

$$
J=\left(R_{P}\right)^{-1} V / L
$$

where $R_{P}$ is the shunt resistivity or parallel resistivity (in units of $\Omega \mathrm{cm}^{2}$ ). Shunt currents are especially relevant for asymmetric devices with a large $V_{\mathrm{bi}}$ since the current density at low voltages is greatly reduced in these devices (as is obvious later in this study).

In principle, the total current density of an asymmetric single-carrier device can be calculated by using a 
combination of Eqs. (1) (with the $V$ - $V_{\mathrm{bi}}$ correction), (4), and (7) if the semiconductor in the device is intrinsic [a symmetric device can likewise, in principle, be calculated using a combination of Eqs. (1) and (6)]. However, this description of the current density does not account for the presence of traps, which have-on numerous occasionsbeen shown to be present in organic semiconductors [14,41].

\section{ANALYTICAL RESULTS}

Reference [40] has shown that the spatial distribution of the equilibrium charge-carrier density of an intrinsic and trap-free single-carrier device $n$ can be described, to a very good approximation, by

$$
n=\frac{2 \pi^{2} \varepsilon_{r} \varepsilon_{0} k_{B} T}{q^{2} L^{2}}\left[\cos ^{2}\left\{\pi\left(\frac{x}{L}-\frac{1}{2}\right)\right\}\right]^{-1},
$$

where $x$ is the distance from the injecting contact. As we show, Eq. (8) precisely describes the charge-carrier density in the middle of the device $x=L / 2$ but produces unphysical

(a)

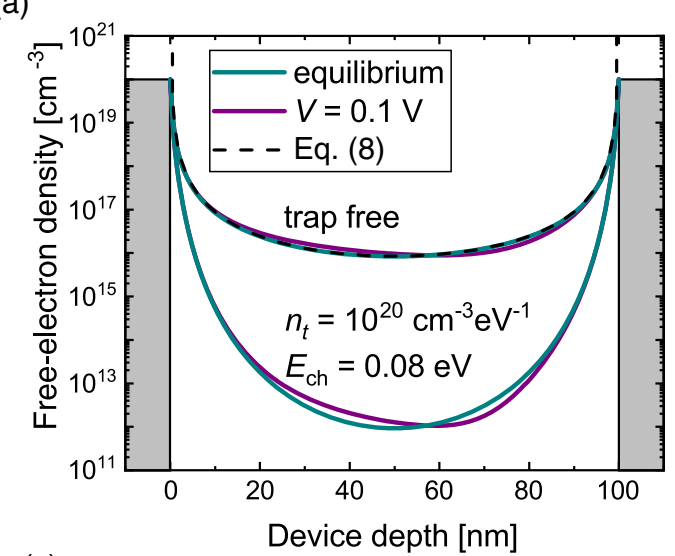

(c)

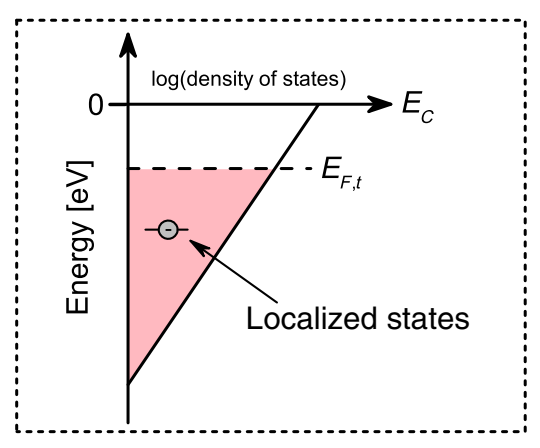

singularities at the contacts $x=0$ and $x=L$, as seen in Fig. 2(a). By taking $x=L / 2$, the equilibrium charge-carrier density in the middle of the device is given by

$$
n_{0}=\frac{2 \pi^{2} \varepsilon_{r} \varepsilon_{0} k_{B} T}{q^{2} L^{2}} .
$$

A comparison of Eq. (9) with numerically calculated values of the electron density is shown in Fig. 2(b) (solid lines).

The arithmetic mean of Eq. (8) $\langle n\rangle=L^{-1} \int_{0}^{L} n d x$ cannot be calculated since the integral does not converge. However, $n^{-1}$ can be integrated, and, thus, the harmonic mean of the charge-carrier density can be written as

$$
\left\langle n_{\text {ana }}\right\rangle=\frac{1}{\frac{1}{L} \int_{0}^{L}(n)^{-1} d x} .
$$

This yields

$$
\left\langle n_{\mathrm{ana}}\right\rangle=\frac{4 \pi^{2} \varepsilon_{r} \varepsilon_{0} k_{B} T}{q^{2} L^{2}},
$$

(b)

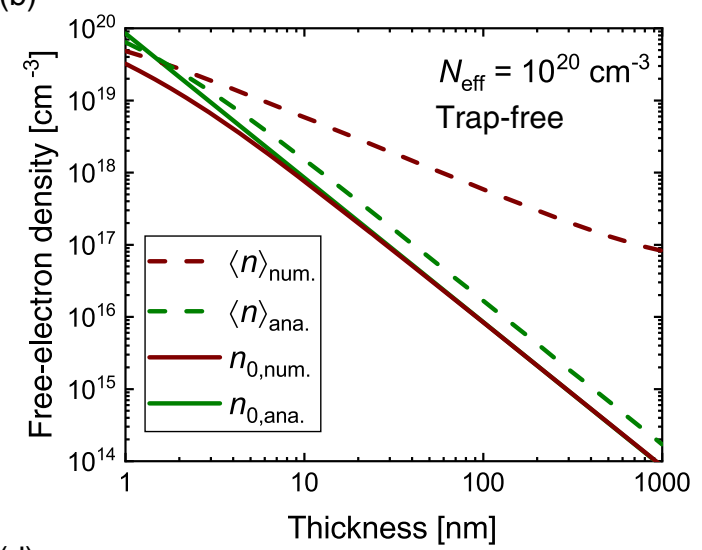

(d)

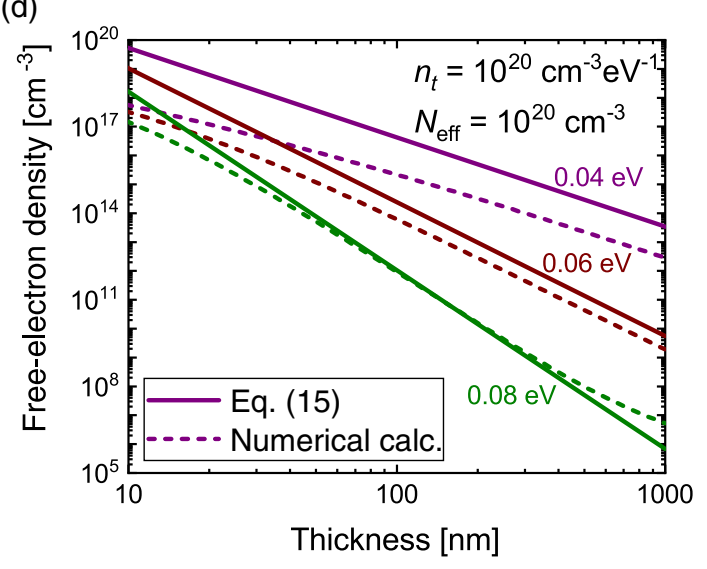

FIG. 2. (a) Electron density as a function of the spatial position of a trap-free device and a device with localized states in the form of exponential tail trap states in comparison with Eq. (8). (b) Average electron densities $n$ of the numerical calculation (red dashed line) and the analytical approximation Eq. (11) (green dashed line) and electron density in the middle of the device $n_{0}$ from the numerical calculation (solid red line) and from Eq. (9) (solid green line). (c) Schematic of the description of the localized states in the band gap. In this case, $T=0 \mathrm{~K}$ is assumed for the representation of the occupied density of states. (d) Electron density in the middle of the device calculated using Eq. (15) (solid lines) and from numerical calculations (dashed lines) when traps are included for three values of $E_{\mathrm{ch}}$ as indicated, $0.04,0.06$, and $0.08 \mathrm{eV}$. 
which is, of course, just $2 n_{0}$. Upon insertion of this chargecarrier density into the drift-current equation $J=q \mu_{n}\langle n\rangle_{\text {ana }}$ $(V / L)$, we obtain the ME equation (6). The ME equation is a very good approximation of the current density in an intrinsic single-carrier device at low voltages. Equation (9) is a very good approximation of the charge-carrier density in the middle of the device at low applied voltages, since the overall shape and magnitude of the charge-carrierdensity profile does not change significantly between 0 and $0.1 \mathrm{~V}$ [see Fig. 2(a)].

It is important to note that the harmonic mean of the charge-carrier density $\langle n\rangle_{\text {ana }}$ does not give the same value of the arithmetic mean of the charge-carrier density of a device calculated using a numerical approach $\langle n\rangle_{\text {num }}$, since the device boundaries are not correctly described in the analytical approximation [since the charge-carrier density at the boundary is set by the effective density of states in the numerical calculations and blows up to infinity in Eq. (8)]. For this reason, the ME equation cannot be directly used to determine the charge-carrier density of the entire device but only in the middle of the device [35]. Figure 2(b) shows that $\langle n\rangle_{\text {num }}>\langle n\rangle_{\text {ana }}$, in general, for an intrinsic and trap-free device.

We now proceed to derive a formula for the currentvoltage response of a single-carrier device in the lowvoltage regime in the presence of traps. The following derivation follows from the assumptions of trapped and free charge-carrier-density statistics in exponential tails given by Mark and Helfrich [8]. For simplicity, the derivation is given for the case of electron transport. The derivation in the case of hole transport is completely analogous.

The density of localized states in the band gap (electron traps) per unit volume and energy interval $h$ far away from the device contacts, i.e., in the middle of the device $(x=L / 2)$, is described by

$$
h=n_{t} \exp \left(\frac{E}{E_{\mathrm{ch}}}\right),
$$

where $n_{t}=N_{t} / E_{\mathrm{ch}}$ is the trap density per unit energy right below the conduction-band edge, and $E$ is the energy measured below the conduction-band edge [note that the conduction-band edge is set to zero $E_{c}=0$, and the energy increases upwards in energy, as shown in Fig. 2(c)]. Given that $N_{t}$ is much larger than the total amount of charge carriers at absolute zero $n_{0}(T=0 \mathrm{~K})$, it is safe to assume that approximately all of these charge carriers will be trapped and will fill the exponential tail states up to a quasiFermi-level, $E_{F, t}$. Within this assumption, we can write

$$
n_{0}=\int_{-\infty}^{E_{F, t}} \frac{N_{t}}{E_{\mathrm{ch}}} \exp \left(\frac{E}{E_{\mathrm{ch}}}\right) d E=N_{t} \exp \left(\frac{E_{F, t}}{E_{\mathrm{ch}}}\right) .
$$

As the temperature is increased $(T>0 \mathrm{~K})$, some charge carriers will escape the traps and will be free to conduct.
Given that the Fermi level governing free charge carriers is more than a few $k_{B} T$ away from the conduction-band edge, we can describe the free charge-carrier density using Boltzmann statistics (again implying $E_{c}=0$ ),

$$
n_{\text {free }}=N_{C} \exp \left(\frac{E_{F}}{k_{B} T}\right),
$$

where $N_{C}$ is the effective density of electron states at the conduction band. Assuming that $E_{F}=E_{F, t}$, which is a fair assumption given that $E_{\mathrm{ch}}>k_{B} T$, we can combine the above two expressions and describe the free charge-carrier density in terms of the total charge-carrier density as

$$
n_{\text {free }}=N_{C} N_{t}^{-l} n_{0}^{l},
$$

where $l=E_{\mathrm{ch}} / k_{B} T$. Figure 2(d) shows a comparison of Eq. (15) with the numerical calculations of the chargecarrier density as the characteristic energy is varied from 0.04 to $0.08 \mathrm{eV}$ (with a fixed $N_{C}$ and $N_{t}$ ). Since the current is governed only by the free charge-carrier density, we can now describe the drift current as

$$
J=q \mu_{n} n_{\text {free }} \frac{V}{L}=q \mu_{n} N_{C}\left\{N_{t}^{-l} n_{0}^{l}\right\} \frac{V}{L} .
$$

Since we are concerned only about the trap density far away from the contacts, we can describe the total chargecarrier density by Eq. (9). We then obtain

$$
J=q^{1-2 l} \mu_{n} N_{C}\left\{\frac{4 \pi^{2} \varepsilon_{r} \varepsilon_{0} k_{B} T}{N_{t}}\right\}^{l} \frac{V}{L^{2 l+1}},
$$

which describes the current density at low applied voltages when some charge carriers are trapped in exponential tails. Note that in this case, we do not need to introduce the concept of an effective mobility since the reduction of free charge carriers is included implicitly through $n_{\text {free }}=N_{C} N_{t}^{-l} n_{0}^{l}$. $\mu_{n}$ is, for that reason, a bandlike mobility. Equation (17) reduces to Eq. (6) in the trap-free limit. The accuracy of estimating trap densities and energies using Eq. (17) in the low-voltage regime is compared to the accuracy of estimating traps with the $\mathrm{MH}$ equation in the intermediate-voltage regime in the Supplemental Material [25]. It is shown that Eq. (17) is more precise in describing charge transport in a semiconductor containing traps (in the investigated parameter space), and it can estimate the characteristic energy and trap density more accurately. The reason why Eq. (17) works especially well is due to the fact that the free charge-carrier density is very well described in the middle of the device using the surprisingly simple trap statistics [see Figs. 2(a) and 2(b)] and the fact that this middle region of the device dominates the ME current.

\section{EXPERIMENTAL RESULTS}

In order to make a comparative evaluation of the different analytical approaches and the numerical drift- 
(a)

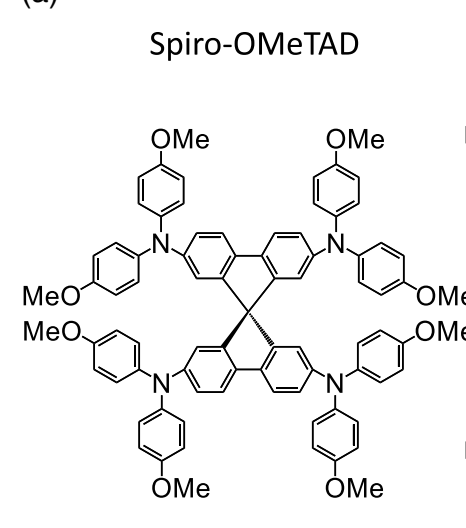

(b)

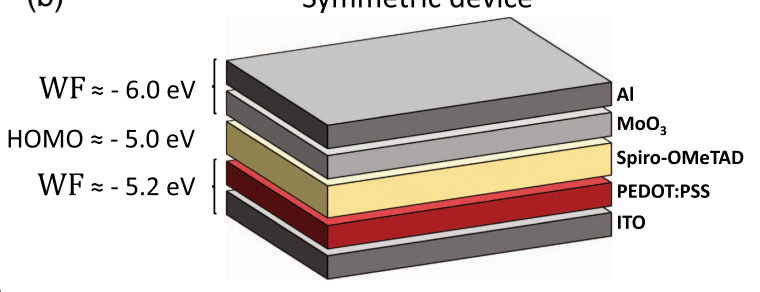

Asymmetric device

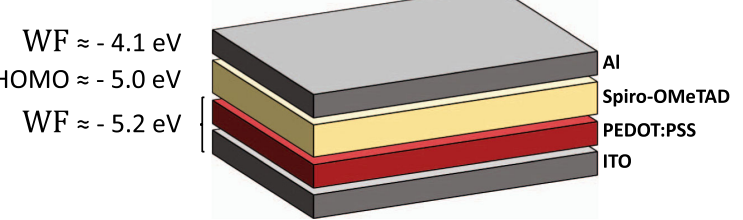

FIG. 3. (a) Molecular structure of spiro-OMeTAD. (b) Symmetric and asymmetric hole-only devices employing $\mathrm{MoO}_{3} / \mathrm{Al}$ and $\mathrm{Al}$ back contacts, respectively (energy levels are taken from the literature). The WFs of $\mathrm{MoO}_{3}$ and PEDOT-PSS will shift to match the HOMO of the organic compound through Fermi-level pinning, resulting in estimated built-in voltages of 0 and $0.9 \mathrm{~V}$, respectively, for the two devices. The energy levels are all given relative to the vacuum level.

diffusion simulation, we prepare and measure a set of holeonly devices based on the small molecule semiconductor spiro-OMeTAD [Fig. 3(a)]. We fabricate both symmetric and asymmetric hole-only devices in order to test the theoretical models over a wide voltage range of $J$ - $V$ data for the different device types. All devices are fabricated using a similar front contact consisting of a thin layer of PEDOT: PSS (approximately $30 \mathrm{~nm}$ ) spin cast on top of a predefined tin-doped indium-oxide- (ITO) covered glass slide. The ITO is cleaned by ultrasonication in acetone and isopropanol for $10 \mathrm{~min}$, respectively, prior to PEDOT:PSS deposition. Thin films of spiro-OMeTAD of varying thicknesses are spin cast from chlorobenzene onto the ITO/PEDOT:PSS contacts under atmospheric conditions. For the asymmetric devices, a back contact consisting of a thick layer of aluminum $(150 \mathrm{~nm})$ is evaporated under high vacuum (of approximately $10^{-6} \mathrm{mbar}$ ). For the symmetric devices, a thin layer of $\mathrm{MoO}_{3}(30 \mathrm{~nm})$ followed by a thick layer of $\mathrm{Al}(150 \mathrm{~nm})$ is used as a back contact. Two types of steady-state $J-V$ experiments are performed: at room temperature and at varying temperature using a cryostat. The current of the samples is recorded using a Keithley SMU 236 in a nitrogen atmosphere for the roomtemperature measurements and in a helium atmosphere for the cryostat-based temperature-dependent measurements. The structures of the studied single-carrier devices are depicted in Fig. 3(b).

Based on the values for the contact WFs shown in Fig. 3(b), one can expect that the $V_{\mathrm{bi}}$ of the so-called symmetric spiro-OMeTAD device is around $1 \mathrm{~V}$, actually rendering the device asymmetric. However, Fermi-level pinning between a metal contact and an organic compound whose HOMO level is shallower than the contact WF will shift this contact WF to match the HOMO level (upon thermodynamic equilibration), rendering both effective WFs to be equal to the HOMO level and the built-in voltage, therefore, to be zero. Again, assuming Fermi-level pinning, the built-in voltage is estimated to be approximately $0.9 \mathrm{~V}$ for the asymmetric device (using a WF value for PEDOT: PSS of $5.2 \mathrm{eV}$ ). However, interfacial states (potentially bearing considerable dipole moments) between the contacts and the organic material, and the reactive nature of aluminum under ambient atmosphere, might give rise to deviations from these estimated $V_{\mathrm{bi}}$ values.

Figure 4(a) shows the experimental $J-V$ curves of a symmetric 200-nm hole-only device and an asymmetric $230-\mathrm{nm}$ device. In contrast to the nonrectifying behavior observed for the current density between the forward and reverse bias in the symmetric device, a significant rectifying behavior is present for the asymmetric device. Since a large asymmetry between the forward- and reverse-bias current is observed, a built-in voltage is present, and the current at low voltage must be given by Eq. (4) [or Eq. (5) given the current is not limited by injection]. The local slope of the $J-V$ curve on a log-log scale can be given by

$$
m=\frac{d \log J}{d \log V}
$$

such that $J \propto V^{m(V)}$. It is seen from the slopes of the $J-V$ curves on a log-log scale in Fig. 4(b) that the forward-bias current for the asymmetric device goes from a linear dependence at low voltages $(m=1)$, to a large peak at intermediate voltages $(m \cong 30)$, and eventually approaches values $(m \cong 3.7)$ which are larger than what is expected from trap-free behavior $(m=2)$ at high voltages [7]. Also, the reverse-bias current is seen to be linear with the voltage over the whole regime for the asymmetric device. From the observation of the linear regime in both the forward- and reverse-bias regime of the symmetric device, it is clear that the low-voltage current cannot be explained by Eq. (4) 
(a)

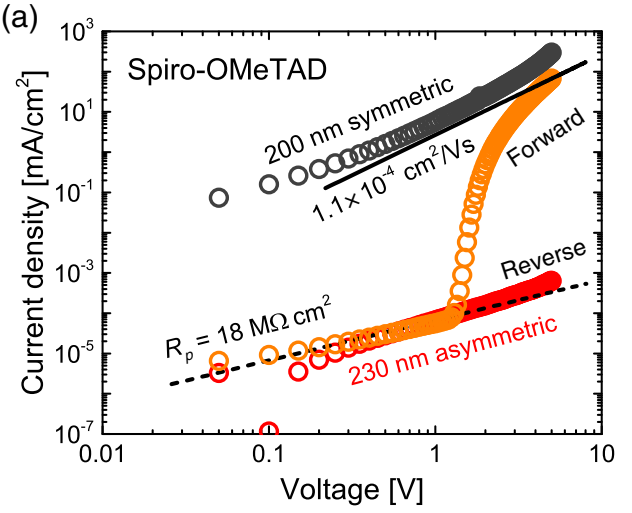

(c)

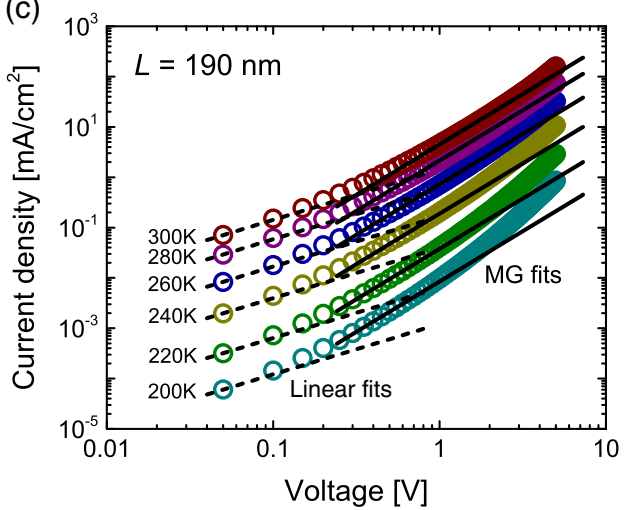

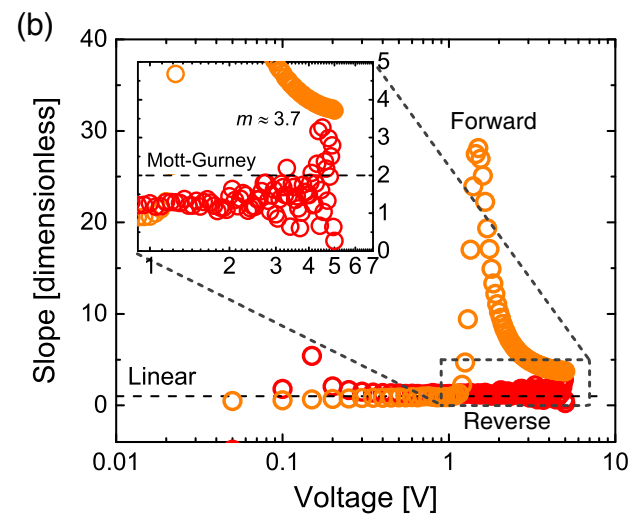

(d)

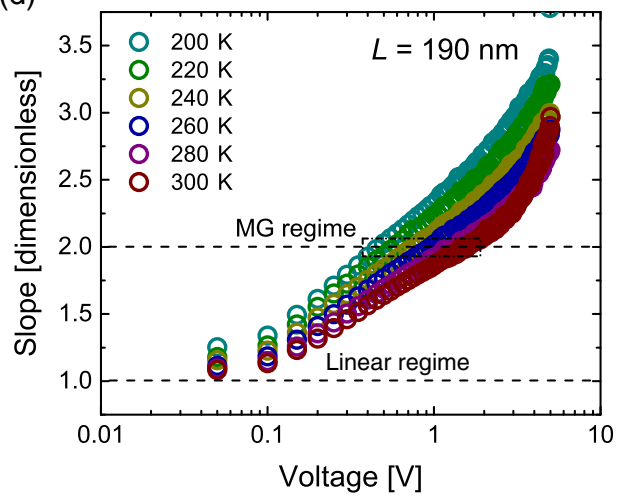

FIG. 4. (a) Forward- and reverse-bias $J-V$ curves obtained from SCLC measurements of symmetric (gray circles) and asymmetric spiroOMeTAD hole-only devices (orange and red circles) of 200 and $230 \mathrm{~nm}$, respectively. Since the forward- and reverse-bias curves overlap for the symmetric device, only the forward-bias curve is shown. Mott-Gurney law forced "fits" and Ohm's law fits are shown as solid and dashed lines, respectively. (b) Plots of $m=(d \log J) /(d \log V)$ of the asymmetric data in (a) against the voltage. (c) $J-V$ curves of symmetric spiroOMeTAD devices at temperatures varying from 200 to $300 \mathrm{~K}$ in steps of $20 \mathrm{~K}$, showing fitting with the Mott-Gurney law [Eq. (1), solid lines] and either the moving-electrode equation [Eq. (6), dashed lines] or Eq. (17). (d) $m-V$ curves of the $J$ - $V$ curves shown in (c). The high-voltage slope values approach a value larger than 2 in both (a) and (c).

alone (Supplemental Material [25] Fig. S1). However, the current can be modeled as a combination of diffusion currents due to a large $V_{\mathrm{bi}}$ and a shunt current [Eq. (7)]. That the current must include a shunt term at low voltages can also be seen from the very sharp onset to the large peak at forward bias (Supplemental Material [25] Fig. S2) along with the large overlap between the forward- and reversebias current at low voltages. Based on this reasoning, the linear currents at very low voltages can be described in both forward and reverse bias using Eq. (7) (dashed lines), and a value for the shunt resistivity of $R_{P}=18 \mathrm{M} \Omega \mathrm{cm}^{2}$ can be extracted. The deviation of the power-law dependence of the $J-V$ curves away from $m=2$ at high voltages [Fig. 4(b)] gives evidence that the charge transport is governed by traps $[6,8]$, as traps in the form of exponential tail states give rise to an increase in the slope away from 2 [Eq. (3) and Supplemental Material [25] Fig. S1].

Since it is quite evident from the slope at high voltages that a proper charge-transport analysis cannot be achieved using the simple MG theory, we carry out numerical fitting using a drift-diffusion solver (see the Supplemental Material for details [25]). To reduce the uncertainty in fitting parameters, especially regarding trap states, we analyze $J-V$ data as a function of both the temperature and semiconductor thickness. Figure 4(c) shows the series from a 190-nm spiro-OMeTAD symmetric hole-only device at temperatures varying from 200 to $300 \mathrm{~K}$ along with linear fits with the ME equation or Eq. (17) (dashed lines) and fits with the MG law (solid lines). $m-V$ curves of the $J$ - $V$ curves in Fig. 4(c) are shown in Fig. 4(d). The linear regime and the apparent Mott-Gurney regime are shown as dashed lines (the linear equations are fitted in the lowvoltage regime, and the MG law is fitted at the point where $m=2$ ). If a fit with the MG law is performed in the trapinfluenced regime, as is sometimes seen in the literature, the values for the effective charge-carrier mobilities between (1-3) $\times 10^{-4} \mathrm{~cm}^{2} /$ Vs for the symmetric devices are obtained (at room temperature). These values are similar to what has been presented in the past using SCLCs [22] [a fit to the asymmetric data shown in Fig. 4(a) gave a mobility with a comparable value of $\left.1.1 \times 10^{-4} \mathrm{~cm}^{2} / \mathrm{Vs}\right]$. The results of the $\mathrm{ME}$ and $\mathrm{MG}$ fits as a function of the temperature are shown in Fig. 6(a).

Figure 5(a) shows the fits from the drift-diffusion simulations to both the forward- and reverse-bias 


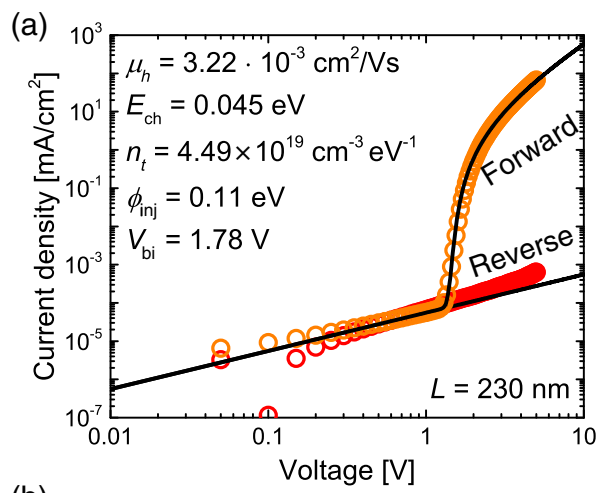

(b)
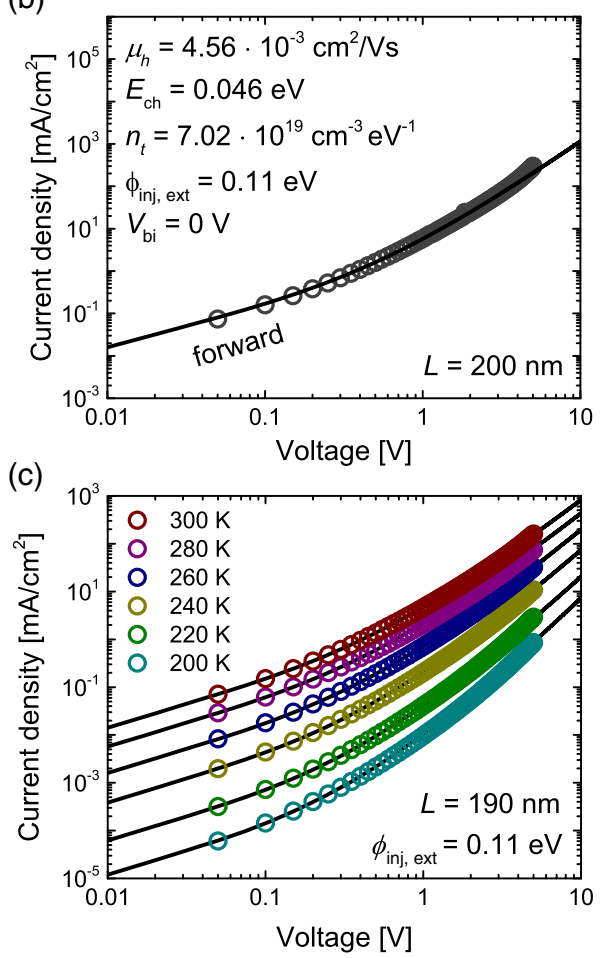

FIG. 5. Numerical fits to SCLC data. (a) Forward- and reversebias current of an asymmetric device, (b) forward-bias current of a symmetric device (the forward- and reverse-bias currents overlap), and (c) forward-bias current of a symmetric device at varying temperatures. Results of the fits in (a) and (b) are shown in the graphs. An injection barrier of $0.11 \mathrm{eV}$ and an exponential tail of trap states are required to consistently fit to the temperature series.

asymmetric device data. Figure 5(b) shows the fits to the forward-bias current of the symmetric device (the forward- and reverse-bias currents overlap). Since the shunt resistivity is determined from a fit with Ohm's law [Fig. 4(a)], this value can be used as input for the drift-diffusion fit along with the measured device thickness. The field-, temperature-, and charge-carrier-densityindependent hole bandlike mobility $\mu_{h}$, characteristic tail energy $E_{\mathrm{ch}}$, tail state density $n_{t}$, and injection and extraction barrier heights $q \phi_{\text {inj }}$ and $q \phi_{\text {ext }}$ are allowed to vary during the fitting process for both the symmetric and asymmetric device data. Figure 5(c) shows the fits using the drift-diffusion model to the temperature series with the inclusion of exponential tails and injection barriers as those obtained in Fig. 5(b). (The fitting results along with additional fits performed on 115- and 290-nm devices are shown in the Supplemental Material [25] Figs. S3-S5. These results are consistent with the results shown in Fig. 5. A deviation from the trend is observed for the 115 -nm device at low temperature, which is assigned to be due to the experimental conditions.] Note that the parameters obtained from this fitting vary slightly with the temperature. The relative insensitivity of the parameters to the temperature indicate that the underlying transport model is valid. However, a moderately good set of fits to the SCLC $J-V$ data can be obtained by making the fit with the constraint that all parameters are completely temperature independent, as shown in the Supplemental Material [25] Figs. S6(b)-S6(d). Whereas the values for the trap characteristics and barrier heights affect the slope of the $J-V$ curves, since no recombination occurs and the measurement is performed under steady-state conditions, $\mu_{h}$ affects only the magnitude of the current density, meaning that the $m-V$ curves can be fitted prior to the $J-V$ curves.

\section{DISCUSSION}

The $V_{\mathrm{bi}}$ for the symmetric device [Fig. 5(b)] is found to be zero, as expected, with barrier heights for both injection and extraction of $0.11 \mathrm{eV}$. The $V_{\mathrm{bi}}$ for the asymmetric device [Fig. 5(a)] is determined to be $1.78 \mathrm{~V}$ with an injection barrier height of $0.11 \mathrm{eV}$. The determined $V_{\mathrm{bi}}$ for the asymmetric device is, therefore, $0.88 \mathrm{~V}$ higher than the value expected from the nominal energy-level offset [Fig. 2(b)]. This is likely due to oxidation of the aluminum contact forming a thin aluminum oxide layer at the contact-semiconductor interface [42]. An underestimation of the $V_{\mathrm{bi}}$ using Eq. (5) is observed [Supplemental Material [25] Figs. S7(b) and S7(c)], since this equation does not account for both traps and injection barrier heights.

Both the symmetric and asymmetric devices show evidence of shallow exponential tails $\left(E_{\mathrm{ch}} \cong 0.045 \mathrm{eV}\right)$ obtained from the drift-diffusion simulations, with trap densities extending from the band edges of $4.49 \times 10^{19}$ and $7.02 \times 10^{19} \mathrm{~cm}^{-3} \mathrm{eV}^{-1}$ for the asymmetric and symmetric device, respectively. The $\mathrm{MH}$ equation (3) gives a much higher estimate of $E_{\mathrm{ch}}\left(J \propto V^{l+1}\right.$ with $\left.l=E_{\mathrm{ch}} / k_{B} T\right)$ [11] of $0.070 \mathrm{eV}$ if fit to the asymmetric device data shown in Fig. 5(a). Note that using the $\mathrm{MH}$ equation to the temperature-dependent data yields a value for the characteristic energy, which decreases with increased temperature [Fig. 4(d)] since the slope decreases. A temperaturedependent characteristic energy is, however, not observed when we analyze the data using the numerical model [Supplemental Material [25] Figs. S3(c), S4(c), and S5(c)].

For the devices in Fig. 4(a), the hole bandlike mobility $\mu_{h}$ is determined to be $3.22 \times 10^{-3}$ and $4.56 \times 10^{-3} \mathrm{~cm}^{2} \mathrm{~V}^{-1} \mathrm{~s}^{-1}$ 


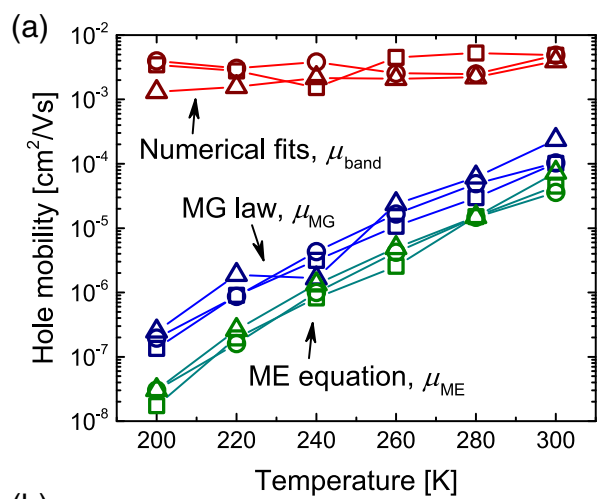

(b)
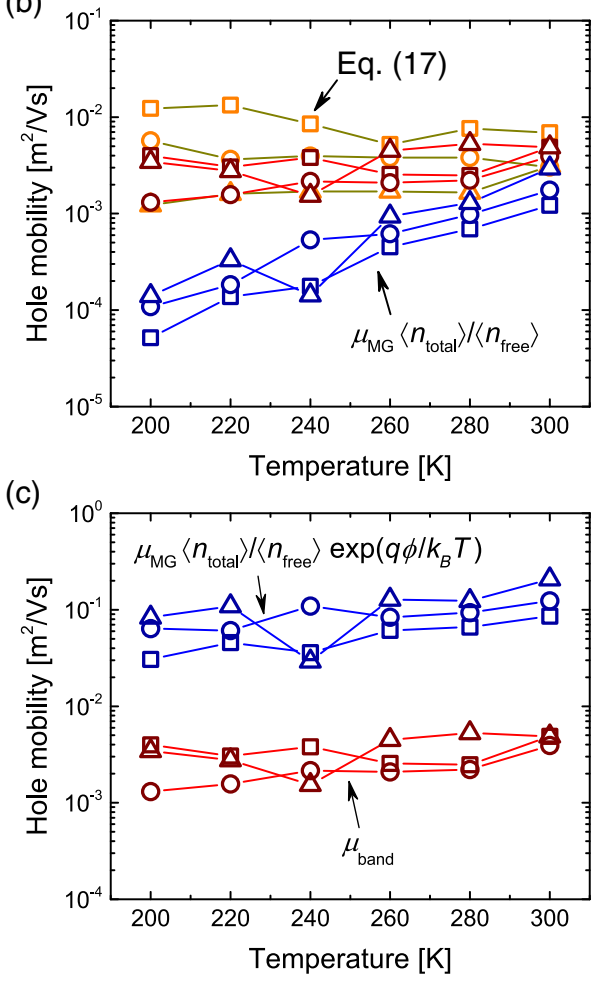

FIG. 6. (a) Resulting mobility values using various techniques [red for numerical fitting, blue for the Mott-Gurney law fitting Eq. (1), and green for the moving-electrode equation fitting Eq. (6)]. The set of various symbols refers to values inferred from devices with different semiconducting layer thicknesses: $290 \mathrm{~nm}$ (squares), $190 \mathrm{~nm}$ (circles) and $115 \mathrm{~nm}$ (triangles), respectively. (b) Comparison of the bandlike mobility with the effective mobility from the MG law modified by the ratio of total to free charge carriers [Supplemental Material [25] Figs. S7(a) and S7 (b)], and with the value estimated using Eq. (17). (c) Comparison of the bandlike mobility with the MG mobility modified for both traps and injection limitation at the metal-semiconductor interfaces. If the MG mobility is used to fit the entire voltage range for the 190-nm device, an apparent field dependence is observed, as shown in the Supplemental Material [25] Fig. S7(d).

for the asymmetric and symmetric devices, respectively, which is orders of magnitude higher than the effective mobility obtained from the MG evaluation [Fig. 6(a)] and an order of magnitude higher than the effective hole mobility reported in the past from SCLC characterization of spiro-OMeTAD [22].

Figure 6(a) compares the hole mobility values obtained from fits with the numerical model to the data [Fig. 5(c)] to mobilities obtained by fitting Eq. (1) or (6) [Fig. 4(c)]. The obtained trap characteristics from the numerical fits are shown in the Supplemental Material [25] Figs. S3-S5. Where a temperature-dependent mobility is obtained using analytical fits with Eqs. (1) and (6), a temperatureindependent bandlike mobility is obtained using numerical fits with the inclusion of traps and the inclusion of injection limitation at the device interfaces. From the numerical model, the obtained charge-carrier mobility at room temperature $(300 \mathrm{~K})$ is found to be more than an order of magnitude higher than the effective mobility and more than 4 orders of magnitude higher at $200 \mathrm{~K}$. While the mobility returned by the numerical model is a bandlike mobility, the mobility returned by the analytical equations represents the bandlike mobility weighted by the effects of trapping and poor charge injection, i.e., an effective mobility. The temperature dependence of the charge transport then originates from injection limitation along with detrapping being a thermally activated process that becomes more difficult at lower temperatures and not from a temperature-dependent bandlike mobility.

Figure 6(b) compares the temperature-independent bandlike mobility with the mobility obtained from Eq. (17) and the effective mobility extracted from fitting with the MG law (modified by the ratio of the arithmetic means of the total to free charge carriers obtained from the drift-diffusion simulations, $\left.\mu_{\mathrm{MG}}\left\langle n_{\text {total }}\right\rangle /\left\langle n_{\text {free }}\right\rangle\right)$. Note that the MG law modification is only possible by simulating the densities of free and trapped holes in the device. Thus, this modification is not available if a purely analytical approach is used. The ratio $\left\langle n_{\text {total }}\right\rangle /\left\langle n_{\text {free }}\right\rangle$ is evaluated at the same applied voltage as the Mott-Gurney law (the voltage at which $m=2$ ) (see the Supplemental Material [25] Fig. S8). Good agreement is found between the mobility obtained from Eq. (17) and the mobility obtained from the numerical fits when the average value for the characteristic energy and trap density from the simulations averaged over all thicknesses and temperatures is used in Eq. (17) $\left(n_{t}=1.31 \times\right.$ $10^{20} \mathrm{~cm}^{-3} \mathrm{eV}^{-1}$ and $E_{\mathrm{ch}}=0.044 \mathrm{eV}$, respectively). Similar to fitting with the MH equation, in order to use Eq. (17) for determining the mobility and trap characteristics, without any prior knowledge of either the mobility or trap characteristics, a thickness and temperature series should be analyzed to reach a convergence of the parameters as shown in Fig. 6(b). However, the much-improved accuracy of determining the bandlike mobility $E_{\mathrm{ch}}$ and $n_{t}$ using Eq. (17) compared to the MH equation is shown in the Supplemental Material [25] Fig. S9. Contrary to the good agreement between Eq. (17) and the numerical calculations, the overall magnitude of the effective mobility from the modified MG law is seen to approach only the bandlike mobility at $300 \mathrm{~K}$. 
Figure 6(c) compares the bandlike mobility with the effective mobility now corrected for both trapping and a term for injection limitation $\mu_{\mathrm{MG}}\left\langle n_{\text {total }}\right\rangle /\left\langle n_{\text {free }}\right\rangle \exp$ $\left(q \phi_{\text {inj }} / k_{B} T\right)$. Interestingly, the temperature independence of this effective charge-carrier mobility is now comparable to that of the bandlike mobility, however, with the overall magnitude being an order of magnitude too high.

From the above discussion, it is evident that a proper (non-ad-hoc) inclusion of traps is important to correctly extract trap characteristics, such as the application of Eq. (17) or the numerical approach. Furthermore, proper inclusion of traps and injection limitation is crucial for fitting to the entire voltage range, i.e., for the correct determination of the bandlike charge-carrier mobility using a numerical drift-diffusion simulator, since the probed current-voltage behavior is affected by these effects at higher voltages simultaneously in a nontrivial manner.

\section{CONCLUSIONS}

By allowing the charge-carrier mobility, trap characteristics, injection barrier heights, and the shunt resistivity to vary, we show that a numerical fit can be obtained to the entire $J-V$ curve measured from both symmetric and asymmetric single-carrier devices made from an organic semiconductor (spiro-OMeTAD). The obtained chargecarrier mobilities and trap densities for both the symmetric and the asymmetric devices agree within a factor of less than 2 across several device thicknesses and over a large range of temperatures. Moreover, the hole bandlike mobility obtained from numerical fitting is more than an order of magnitude higher $\left(4.56 \times 10^{-3} \mathrm{~cm}^{2} \mathrm{~V}^{-1} \mathrm{~s}^{-1}\right)$ than the effective hole mobility determined using the Mott-Gurney law $\left(2.30 \times 10^{-4} \mathrm{~cm}^{2} \mathrm{~V}^{-1} \mathrm{~s}^{-1}\right)$ at room temperature $(300 \mathrm{~K})$ and more than 4 orders of magnitude at $200 \mathrm{~K}$. We further show that while simple analytical equations require a temperature-dependent mobility to achieve fits to the experimental data, the use of either an analytical model that accounts for traps-such as the analytical model presented in this paper-or a numerical model give rise to a temperatureindependent bandlike mobility while simultaneously yielding information about trap characteristics and injection statistics. Our analysis and results highlight the importance of either showing and using a numerical model to fit to the entire $J-V$ curve while accounting for traps, the built-in voltage, and injection limitation, or to use a more sophisticated analytical model which can correctly account for traps, such as the model presented herein.

\section{ACKNOWLEDGMENTS}

J. A. R., S. A. H., and J. N. thank the Engineering and Physical Sciences Research Council (EPSRC Grants No. EP/K030671/1 and No. EP/K010298/1) and the Doctoral Training Centre for Plastic Electronics (Grant No. EP/G037515) for funding. X. S. and J. N. thank the
Engineering and Physical Sciences Research Council (via Grants No. EP/M025020/1 and No. EP/K029843/1). T. K. acknowledges funding via an Imperial College Junior Research Fellowship.

[1] H. Sirringhaus, P. J. Brown, R. H. Friend, M. M. Nielsen, K. Bechgaard, and A. J. H. Spiering, Two-dimensional charge transport in self-organized, high-mobility conjugated polymers, Nature (London) 401, 685 (1999).

[2] H. Bässler and A. Köhler, Charge transport in organic semiconductors, Top. Curr. Chem. 312, 1 (2012).

[3] J. Dacuña, W. Xie, and A. Salleo, Estimation of the spatial distribution of traps using space-charge-limited current measurements in an organic single crystal, Phys. Rev. B 86, 115202 (2012).

[4] R. Noriega, J. Rivnay, K. Vandewal, F. P. V. Koch, N. Stingelin, P. Smith, M. F. Toney, and A. Salleo, A general relationship between disorder, aggregation and charge transport in conjugated polymers, Nat. Mater. 12, 1038 (2013).

[5] N. F. Mott and R. W. Gurney, Electronic Processes in Ionic Crystals (Oxford University Press, New York, 1940).

[6] A. Rose, Space-charge-limited currents in solids, Phys. Rev. 97, 1538 (1955).

[7] M. A. Lampert, Simplified theory of space-charge-limited currents in an insulator with traps, Phys. Rev. 103, 1648 (1956).

[8] P. Mark and W. Helfrich, Space-charge-limited currents in organic crystals, J. Appl. Phys. 33, 205 (1962).

[9] P. H. Nguyen, S. Scheinert, S. Berleb, W. Brutting, and G. Paasch, The influence of deep traps on transient currentvoltage characteristics of organic light-emitting diodes, Org. Electron. 2, 105 (2001).

[10] P. De Bruyn, A. H. P. Van Rest, G. A. H. Wetzelaer, D. M. De Leeuw, and P. W. M. Blom, Diffusion-Limited Current in Organic Metal-Insulator-Metal Diodes, Phys. Rev. Lett. 111, 186801 (2013).

[11] J. Fischer, W. Tress, H. Kleemann, J. Widmer, K. Leo, and M. Riede, Exploiting diffusion currents at Ohmic contacts for trap characterization in organic semiconductors, Org. Electron. 15, 2428 (2014).

[12] H. T. Nicolai, M. M. Mandoc, and P. W. M. Blom, Electron traps in semiconducting polymers: Exponential versus Gaussian trap distribution, Phys. Rev. B 83, 195204 (2011).

[13] J. Dacuña and A. Salleo, Modeling space-charge-limited currents in organic semiconductors: Extracting trap density and mobility, Phys. Rev. B 84, 195209 (2011).

[14] H. T. Nicolai, M. Kuik, G. a. H. Wetzelaer, B. de Boer, C. Campbell, C. Risko, J. L. Brédas, and P. W. M. Blom, Unification of trap-limited electron transport in semiconducting polymers, Nat. Mater. 11, 882 (2012).

[15] F. Liu, A. Massé, P. Friederich, F. Symalla, R. Nitsche, W. Wenzel, and P. A. Bobbert, $A b$ initio modeling of steady-state and time-dependent charge transport in holeonly $\alpha$-NPD devices, Appl. Phys. Lett. 109, 243301 (2016).

[16] F. Liu, H. van Eersel, B. Xu, J. G. E. Wilbers, M. P. de Jong, W. G. van der Wiel, P. A. Bobbert, and R. Coehoorn, Effect of Coulomb correlation on charge transport in disordered organic semiconductors, Phys. Rev. B 96, 205203 (2017). 
[17] W. F. Pasveer, J. Cottaar, C. Tanase, R. Coehoorn, P. A. Bobbert, P. W. M. Blom, D. M. de Leeuw, and M. A. J. Michels, Unified Description of Charge-Carrier Mobilities in Disordered Semiconducting Polymers, Phys. Rev. Lett. 94, 206601 (2005).

[18] J. C. Blakesley, H. S. Clubb, and N. C. Greenham, Temperature-dependent electron and hole transport in disordered semiconducting polymers: Analysis of energetic disorder, Phys. Rev. B 81, 045210 (2010).

[19] J. M. Montero and J. Bisquert, Trap origin of fielddependent mobility of the carrier transport in organic layers, Solid State Electron. 55, 1 (2011).

[20] R. C. I. MacKenzie, T. Kirchartz, G. F. A. Dibb, and J. Nelson, Modeling nongeminate recombination in P3HT: PCBM solar cells, J. Phys. Chem. C 115, 9806 (2011).

[21] J. M. Frost, J. Kirkpatrick, T. Kirchartz, and J. Nelson, Parameter free calculation of the subgap density of states in poly (3-hexylthiophene), Faraday Discuss. Chem. Soc. 174, 255 (2014).

[22] D. Poplavskyy and J. Nelson, Nondispersive hole transport in amorphous films of methoxy-spirofluorene-arylamine organic compound, J. Appl. Phys. 93, 341 (2003).

[23] R. W. Smith and A. Rose, Space-charge-limited currents in single crystals of cadmium sulfide, Phys. Rev. 97, 1531 (1955).

[24] T. Kirchartz, Influence of diffusion on space-charge-limited current measurements in organic semiconductors, Beilstein J. Nanotechnol. 4, 180 (2013).

[25] See Supplemental Material at http://link.aps.org/ supplemental/10.1103/PhysRevApplied.9.044017 for additional simulation results and the derivations of key equations.

[26] A. M. Nardes, M. Kemerink, M. M. De Kok, E. Vinken, K. Maturova, and R. A. J. Janssen, Conductivity, work function, and environmental stability of PEDOT:PSS thin films treated with sorbitol, Org. Electron. 9, 727 (2008).

[27] Y. Kim, A. Ballantyne, J. Nelson, and D. D. C. Bradley, Conductivity, work function, and environmental stability of PEDOT: PSS thin films treated with sorbitol, Org. Electron. 10, 205 (2009).

[28] M. Kröger, S. Hamwi, J. M. Philips, T. Riedl, and W. Kowalsky, Role of the deep-lying electronic states of in the enhancement of hole-injection in organic thin films, Appl. Phys. Lett. 95, 123301 (2009).
[29] M. T. Greiner, L. Chai, M. G. Helander, W. Tang, and Z. Lu, Transition metal oxide work functions: The influence of cation oxidation state and oxygen vacancies, Adv. Funct. Mater. 22, 4557 (2012).

[30] P. N. Murgatroyd, Theory of space-charge-limited current enhanced by Frenkel effect, J. Phys. D 3, 151 (1970).

[31] J. C. Blakesley, F. a. Castro, W. Kylberg, G. F. a. Dibb, C. Arantes, R. Valaski, M. Cremona, J. S. Kim, and J. S. Kim, Towards reliable charge-mobility benchmark measurements for organic semiconductors, Org. Electron. 15, 1263 (2014).

[32] Z. He, C. Zhong, X. Huang, W.-Y. Wong, H. Wu, L. Chen, $\mathrm{S}$. $\mathrm{Su}$, and Y. Cao, Simultaneous enhancement of opencircuit voltage, short-circuit current density, and fill factor in polymer solar cells, Adv. Mater. 23, 4636 (2011).

[33] A. A. Grinberg and S. Luryi, Space-charge-limited current and capacitance in double-junction diodes, J. Appl. Phys. 61, 1181 (1987).

[34] G. A. H. Wetzelaer and P. W. M. Blom, Ohmic current in organic metal-insulator-metal diodes revisited, Phys. Rev. B 89, 241201 (2014).

[35] J. A. Röhr, T. Kirchartz, and J. Nelson, On the correct interpretation of the low voltage regime in intrinsic singlecarrier devices, J. Phys. Condens. Matter 29, 205901 (2017).

[36] P. E. Schmidt and H. E. Henisch, Drift-diffusion theory of symmetrical double-junction diodes, Solid State Electron. 25, 1129 (1982).

[37] J. G. Simmons, Richardson-Schottky Effect in Solids, Phys. Rev. Lett. 15, 967 (1965).

[38] R. de Levie, N. G. Seidah, and H. Moreia, Transport of ions of one kind through thin membranes, J. Membr. Biol. 10, 171 (1972).

[39] J. G. Simmons, Theory of metallic contacts on high resistivity solids-I. Shallow traps, J. Phys. Chem. Solids 32, 1987 (1971).

[40] S. L. M. van Mensfoort and R. Coehoorn, Effect of Gaussian disorder on the voltage dependence of the current density in sandwich-type devices based on organic semiconductors, Phys. Rev. B 78, 085207 (2008).

[41] T. Kirchartz, B. E. Pieters, J. Kirkpatrick, U. Rau, and J. Nelson, Recombination via tail states in polythiophene: Fullerene solar cells, Phys. Rev. B 83, 115209 (2011).

[42] R. Michel, J. Gastaldi, C. Allasia, and C. Jourdan, Initial interaction of oxygen with aluminium single crystal faces: A LEED, AES and work function study, Surf. Sci. 95, 309 (1980). 